\title{
Characterization of the kidney transcriptome of the South American olive mouse Abrothrix olivacea
}

\author{
Facundo M Giorello ${ }^{1 *}$, Matias Feijoo ${ }^{1}$, Guillermo D'Elía², Lourdes Valdez², Juan C Opazo², Valeria Varas², \\ Daniel E Naya ${ }^{1}$ and Enrique P Lessa ${ }^{1}$
}

\begin{abstract}
Background: The olive mouse Abrothrix olivacea is a cricetid rodent of the subfamily Sigmodontinae that inhabits a wide range of contrasting environments in southern South America, from aridlands to temperate rainforests. Along its distribution, it presents different geographic forms that make the olive mouse a good focal case for the study of geographical variation in response to environmental variation. We chose to characterize the kidney transcriptome because this organ has been shown to be associated with multiple physiological processes, including water reabsorption.

Results: Transcriptomes of thirteen kidneys from individuals from Argentina and Chile were sequenced using Illumina technology in order to obtain a kidney reference transcriptome. After combining the reads produced for each sample, we explored three assembly strategies to obtain the best reconstruction of transcripts, TrinityNorm and DigiNorm, which include its own normalization algorithms for redundant reads removal, and Multireads, which simply consist on the assembly of the joined reads. We found that Multireads strategy produces a less fragmented assembly than normalization algorithms but recovers fewer number of genes. In general, about 15000 genes were annotated, of which almost half had at least one coding sequence reconstructed at $99 \%$ of its length. We also built a list of highly expressed genes, of which several are involved in water conservation under laboratory conditions using mouse models.
\end{abstract}

Conclusion: Based on our assembly results, Trinity's in silico normalization is the best algorithm in terms of cost-benefit returns; however, our results also indicate that normalization should be avoided if complete or nearly complete coding sequences of genes are desired. Given that this work is the first to characterize the transcriptome of any member of Sigmodontinae, a subfamily of cricetid rodents with about 400 living species, it will provide valuable resources for future ecological and evolutionary genomic analyses.

Keywords: Abrothrix olivacea, Abrotrichini, Cricetidae, Sigmodontinae, Muroidea, RNA-Seq, Gene expression, De novo assembly, Normalization methods

\section{Background}

The olive mouse Abrothrix olivacea [1] is a cricetid rodent of the subfamily Sigmodontinae, one of the largest mammalian subfamilies with about 400 species and 86 living genera [2,3]. The olive mouse is distributed along Chile and Argentinean Patagonia, from $18^{\circ} \mathrm{S}$ to $55^{\circ} \mathrm{S}$ latitude [4], extending for over $1000 \mathrm{~km}$ latitudinally, and encompassing a great variety of environments: coastal deserts in the north, Mediterranean scrubs in central Chile, Valdivian

\footnotetext{
* Correspondence: fagire@gmail.com

'Departamento de Ecología y Evolución, Facultad de Ciencias, Universidad de la República, Montevideo, Uruguay

Full list of author information is available at the end of the article
}

and Magallanic forests through the south of Chile and Argentina and Patagonian steppe towards the Atlantic coast. A. olivacea must withstand the arid Chilean north and the Patagonia steppe, as well as the Valdivian rain forest with $2700 \mathrm{~mm}$ or more of annual rainfall [5]. Given the striking biotic and abiotic differences among these environments, differences in thermoregulation and osmoregulation, among other physiological traits, are expected to occur. Higher tolerance to water shortage in populations from xeric habitat has already been demonstrated [6]. On the basis of variation in morphology, coloration patterns, and more recently DNA sequence data $[4,7]$, many $A$. olivacea subspecies have been described and at 
least two phylogeographic breaks have been found along its distribution [8]. All these characteristics make A. olivacea a good focal case for the study of geographical variation in response to environmental variation.

High-throughput sequencing (HTS) [9] has a wide range of applications, from clinical [10] to functional studies in genomics [11], molecular ecology [12], and microbial diversity [13]. Recently, HTS has also been used to characterize transcriptomes of a growing number of nonmodel species (e.g. [14-17]). RNA-seq is a cost-effective way to obtain large amounts of coding sequences and information about gene expression levels [18]. The goal of covering entire genome or transcriptomes, along with the reduction of the HTS costs [9], has motivated digital normalization strategies [19] to systematize the increasing but uneven coverage in shotgun sequencing datasets. Normalization methods estimate the read abundance, regardless of a reference, using the k-mer median abundance of that read and then decides whether to reject or accept it based on the chosen coverage value $[19,20]$. In this manner, normalization algorithms remove redundant reads but also greatly reduce the total number of k-mers by discarding the majority of the erroneous ones. For example, with a sequencing base error rate of 1 bp per 100 bp sequenced [9], k erroneous k-mers will be produced, being $\mathrm{k}$ equal to $\mathrm{k}$-mers size. This data and error reduction notably decreases the computational requirements for de novo assembly.

In this study, we adopted paired-end Illumina sequencing to characterize the kidney transcriptome of $A$. olivacea. We chose kidney because of its association with multiple physiological processes, including water conservation [21] and nutrition [22]. This transcriptome will serve as a reference for comparative studies of geographical variation within this species, as well as for other studies on the diverse sigmodontine rodents. More than 800 million (M) reads were generated for 13 kidney transcriptomes of individuals sampled across Chile and Argentina. We explored various normalization strategies in order to obtain the best transcripts reconstruction and identify the most expressed genes. This is the first report of a sigmodontine transcriptome.

\section{Results}

\section{Transcriptome sequencing and assembly}

Transcriptome sequencing of 13 libraries using Illumina yielded a total of $\sim 87 \mathrm{~Gb}$ of data, formed by $\sim 430 \mathrm{M}$ of paired reads with an average length of $101 \mathrm{bp}$ (Additional file 1: Table S1). Trimming of low quality bases from the 3 ' end, prior to Trinity [23] de novo assembly, reduced average read length to $83 \mathrm{bp}$. The number of reconstructed contigs per library ranged from 62,499 to 120,209; with average length ranging from 972 to 1174 bp and median from 488 to 585 bp (Table 1). Detailed results for each library are shown in Additional file 1: Table S2.

To obtain a good reference transcriptome, we also explored three strategies: (i) combining reads of all libraries (Multireads), (ii) Trinity's in silico normalization (TrinityNorm) [20], and (iii) digital normalization (DigiNorm) [19]. The last two strategies involve, in order to improve assembly efficiency from high coverage sequencing datasets, the deletion of redundant reads, ideally without harming the quality of the final reconstructed genes. Of these two, TrinityNorm was more severe than DigiNorm in reducing the total number of paired-ends reads from $\sim 430 \mathrm{M}$ to $\sim 22 \mathrm{M}$ vs. $\sim 50 \mathrm{M}$ (Table 1 ). Meanwhile, digital normalization was faster than in silico Trinity normalization: 9 hours vs. 14 hours.

As expected, the Multireads strategy led to a far more time consuming and computationally demanding assembly than either of the normalization methods, being five and over nine times slower than the assembly from DigiNorm and Trinity, respectively (Table 1). Also, the average and median lengths of reconstructed contigs from the Multireads data set were smaller than the assembled contigs from normalized reads, with 1,060 and 443 bp for mulitreads, 1,210 and 575 bp for TrinityNorm, and 1,269 and 696 bp for DigiNorm. These results are consistent with the distribution of the contigs, where almost half $(46 \%)$ of the reconstructed contigs from the Multireads strategy were between 200 and 400 bp (Additional file 1: Table S3). On the other hand, the Multireads strategy reconstructed the longest contigs (Additional file 1: Table S3) with 4,212 above $6,400 \mathrm{bp}$. TrinityNorm and Diginorm reconstructed only 3,073 and 2,726 of contigs above this length, respectively.

The two normalization strategies produced similar assembly results in terms of average and median length of contigs, with a small advantage for DigiNorm values, but they significantly differed in the number of contigs assembled, DigiNorm assembled 85,902 more contigs than TrinityNorm and 87,013 more than the Multireads strategy (Table 1).

\section{Gene annotation and evaluation of reconstructed coding sequences}

Annotation was based on BLASTX searches against: (i) OMA browser mouse protein database, which contains the protein isoforms of Mus musculus genes [24] and (ii) NCBI non-redundant vertebrate protein database. For the two databases the same e-value threshold of $1 \mathrm{e}-10$ was set. For the Multireads, TrinityNorm and DigiNorm strategies, each assembled transcript was also analyzed through the Pfam database [25] using HMMER [26,27] for proteins domain identification. A file summarizing the Pfam and BLASTX results for each of the three strategies is available as Additional file 2. 
Table 1 Main assembly metrics for the three assembly strategies and individual libraries

\begin{tabular}{|c|c|c|c|c|c|}
\hline & \multicolumn{2}{|c|}{ Range (of individual libraries) ${ }^{a}$} & \multirow[t]{2}{*}{ Multireads } & \multirow[t]{2}{*}{ TrinityNorm } & \multirow[t]{2}{*}{ DigiNorm } \\
\hline & $\min$ & $\max$ & & & \\
\hline Reads & 27041064 & 42477318 & 430525978 & 21757448 & 50557782 \\
\hline Total contigs & 62499 & 120209 & 275903 & 277014 & 362916 \\
\hline Max contig length & 9942 & 15496 & 20648 & 19625 & 15961 \\
\hline Min contig length & 201 & 201 & 201 & 201 & 201 \\
\hline Average length & 972 & 1174 & 1060 & 1210 & 1269 \\
\hline Median length & 488 & 585 & 443 & 575 & 696 \\
\hline Running time (hours) & $\mathrm{n} / \mathrm{a}$ & $\mathrm{n} / \mathrm{a}$ & 94 (12 threads) & 10 (12 threads) & 19 (12 threads) \\
\hline Normalization time (hours) & $\mathrm{n} / \mathrm{a}$ & $\mathrm{n} / \mathrm{a}$ & $\mathrm{n} / \mathrm{a}$ & 14 (1 thread) & 9 (1 thread) \\
\hline
\end{tabular}

a"Range (of individual libraries)" shows for each row the maximum and minimum value found among the 13 individual libraries of kidney transcriptome of the olive mouse Abrothrix olivacea.

The maximum number of mouse genes annotated within a particular library was 12,988 from the significant hits of 55,332 contigs of the 120,209 assembled (Table 2 and specimen PPA 443 library in Additional file 1: Table S2 and Table S4a). The union of the 13 individual BLASTX runs only added 1,630 significant hits $(14,618$ in total), indicating the high level of redundant information across libraries. On the other hand, when using the extensive non-redundant vertebrate database as reference, the maximum number of contigs annotated within a single library was 58,404, 3072 contigs more than with the OMA database (Additional file 1: Table S4b). Detailed results for each library are shown in Additional file 1: Table S4. Hereafter we present the results based only on mouse proteins from OMA. This database allow us to count the number of genes and their corresponding reconstructed coding sequences (CDS) and obtain an upper bound estimation of genes orthologous with mouse.

Of the 14,618 mouse genes annotated through the union of all libraries, almost one half $(7,060)$ had at least one putative CDS reconstructed at $>99 \%, 9,290$ at $>90 \%$, and 10,104 at $>80 \%$, of the total expected length. More importantly, in total, 9,434 distinct mouse isoforms of the $24,338(\sim 39 \%)$ available at OMA browser were almost fully reconstructed (>90\%) for A. olivacea (Table 2 ).

Among the three strategies carried out to obtain a reference transcriptome, Multireads reached the lowest number of mouse genes, 14,788; meanwhile TriniNorm and DigiNorm, reached 15,077 and 15,095 respectively. Despite having found the lowest number of genes, the Multireads strategy performed best at reconstructing coding sequences, obtaining similar values to those gathered through the union of the single libraries, with 7053 distinct mouse coding sequences reconstructed at $>99 \%$, 9,543 at $>90 \%$, and 10,480 at $>80 \%$ (Table 2). With regard to genes, of the 14,788 annotated by the Multireads alternative, $47 \%$ had at least one CDS fully (>99\%) reconstructed, clearly surpassing the $39 \%$ and $34 \%$ of TrinityNorm and DigiNorm respectively (Figure 1). Between the two normalization strategies, TrinityNorm

Table 2 Gene annotation and coding sequences reconstruction using BLASTX to OMA browser mouse protein database

\begin{tabular}{|c|c|c|c|c|c|c|c|}
\hline & \multirow{2}{*}{$\begin{array}{c}\text { Minimum } \\
\% \text { of CDS } \\
\text { reconstructed }\end{array}$} & \multicolumn{2}{|c|}{ Range (of individual libraries) } & \multirow[t]{2}{*}{ DigiNorm } & \multirow[t]{2}{*}{ Multireads } & \multirow[t]{2}{*}{ TrinityNorm } & \multirow{2}{*}{$\begin{array}{c}\text { Library } \\
\text { union }\end{array}$} \\
\hline & & $\min$ & $\max$ & & & & \\
\hline Genes & \multirow{2}{*}{99} & $3090(3102)$ & $5021(5037)$ & $5211(5245)$ & 7017 (7053) & $5895(5926)$ & $7060(7131)$ \\
\hline Contigs & & 4371 & 7763 & 15815 & 14881 & 13671 & $\mathrm{n} / \mathrm{a}$ \\
\hline Genes & \multirow{2}{*}{90} & $4534(4557)$ & $6882(6916)$ & $7354(7436)$ & 9467 (9543) & $8252(8325)$ & $9290(9434)$ \\
\hline Contigs & & 6861 & 11361 & 26904 & 22137 & 21825 & $\mathrm{n} / \mathrm{a}$ \\
\hline Genes & \multirow{2}{*}{80} & $5706(5745)$ & $8636(8636)$ & $8530(8665)$ & $10377(10480)$ & $9347(9467)$ & $10104(10367)$ \\
\hline Contigs & & 5422 & 14129 & 36814 & 26745 & 28270 & $\mathrm{n} / \mathrm{a}$ \\
\hline Genes & \multirow{2}{*}{50} & 8025 (8138) & 9905 (10089) & $12261(12610)$ & $12587(12818)$ & $12498(12769)$ & $11874(12544)$ \\
\hline Contigs & & 14168 & 25674 & 76156 & 40607 & 51140 & $\mathrm{n} / \mathrm{a}$ \\
\hline Total genes & & $11564(11941)$ & $12988(13434)$ & 15095 (15717) & 14788(15204) & 15077 (15605) & 14618 (15772) \\
\hline Total contigs & & 32934 & 55332 & 157390 & 70380 & 100786 & $\mathrm{n} / \mathrm{a}$ \\
\hline
\end{tabular}

The first value indicates the number of mouse genes found (for which at least one coding sequence was reconstructed). Values in parenthesis are the number of distinct coding sequences reconstructed at each level. The row corresponding to "contigs" indicates the number of contigs that reconstructed coding sequences (CDS) at each level. 


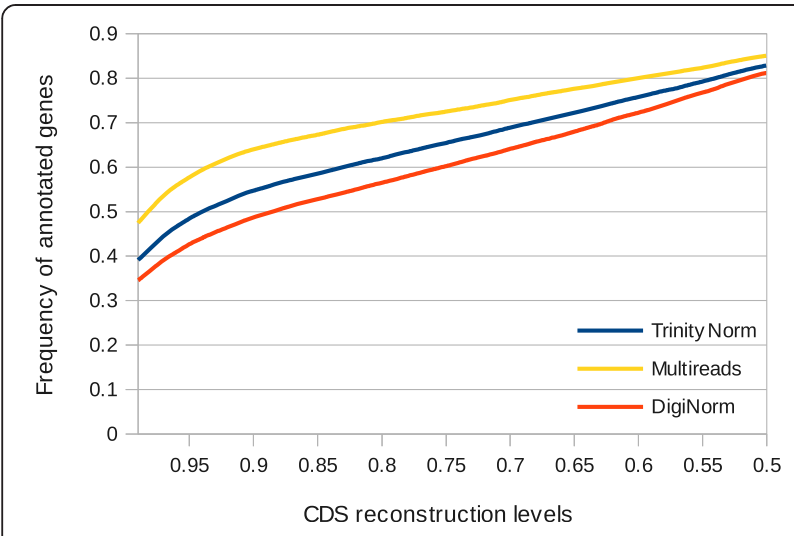

Figure 1 Frequency of genes annotated for Abrothrix olivacea at corresponding CDS reconstruction levels. Frequency of genes annotated per total number of genes were calculated for each strategy (see text) and for the corresponding mouse coding sequences (CDS) reconstruction level. The CDS reconstruction intervals are of 0.01 percent. Only the largest reconstructed CDS for each gene are taken into account.

outperformed DigiNorm at each reconstruction level in terms of numbers of mouse genes found and percentage of coding sequence reconstructed (Table 2 and Figure 1). On the other hand, the assembly from DigiNorm had more contigs at each level of reconstruction and also more contigs with at least one Pfam domain, followed by TriniNorm and the Multireads strategies (Table 2 and Additional file 1: Table S5). This is expected if those contigs represent distinct fragments of the same coding sequence or if they are isotigs (overlapping contigs) representing (ideally) distinct isoforms. However, when the number of potential isoforms from Trinity assembly were inferred and counted (see methods), the average number of alternative reconstructions per contig was 4.9 for DigiNorm and only 2.9 for the Multireads strategy (data not shown). Thus, those contigs are alternative reconstruction (isotigs) representing (possibly) isoforms and not subfragments of a given reference.

\section{Functional annotation}

For the functional annotation of the transcriptome, we selected the genes found by TrinityNorm. This strategy was the one with the best tradeoff between CDS reconstructed, genes found, and computational speed. To this end, the Database for Annotation, Visualization and Integrated Discovery (DAVID) [28], was used to classify them with Gene Ontology (GO) terms.

For the 15,077 genes found by TrinityNorm strategy, 9,793 GO terms were categorized in Biological Processes, 9,486 in Molecular function and 8,978 in Cellular Components. Most genes at Biological Processes belong either to "Regulation of transcription" (1,726), "Transcription" $(1,441)$ and to "Regulation of RNA metabolic process" (1,093) (Figure 2). Likewise, the Molecular
Function category subdivided annotated sequences into "ion binding" (3,234), "cation binding" $(3,201)$, and "metal ion binding" $(3,172)$ as the most represented (Figure 2). Within the category Cellular Component, the three principal groups were: "intrinsic to membrane" $(3,667)$, "integral to membrane" $(3,506)$ and "plasma membrane" $(2,167)$ (Figure 2).

\section{The most expressed genes}

To determine the most expressed genes in the A. olivacea kidney transcriptome, TPM (Transcripts Per Million) expression values were calculated for each single library with RSEM software [29]. For this purpose, a set with 5\% of most expressed genes ( 600 genes) for each of the 13 transcriptomes was identified; these were cross searched to identify those genes common to all libraries. Two hundred eighty-three genes resulted to be present in all libraries (Additional file 3: Table S5). The average TPM values ranged from 333 to 17,798 (Additional file 3: Table S5). Five genes that showed the highest average TPM values were: predicted gene 4076 (possibly a NADHubiquinone oxidoreductase) (ENSMUSG00000096449), glutathione peroxidase 3 (ENSMUSG00000018339), ferritin heavy chain 1 (ENSMUSG00000024661), hemoglobin beta adult major chain (ENSMUSG00000052305), and phosphoenolpyruvate carboxykinase 1 cytosolic (ENSMU G000000027513) (Additional file 3: Table S5).

GO terms for the 283 genes obtained from DAVID showed that the most enriched terms among the three domains using the TrinityNorm genes list as background were: "hydrogen ion transporting ATP synthase activity, rotational mechanism" (17.4 Fold Enrichment, domain: molecular function), "proton-transporting ATP synthase complex" (25.4 Fold Enrichment, cellular component) and "mitochondrial ATP synthesis coupled electron transport" (25.3 Fold Enrichment, biological process) (Additional file 3: Table S6).

Subsequently, these 283 genes were cross-checked with a list obtained from Pradervand et al. [30], who enumerated the most expressed genes in the distal part of the mouse renal tubule using microarrays. Seventeen genes resulted to be in common (Additional file 3: Table S5 in bold): two transcription factors, one small GTPase, eight transporters and channels, and six cytoskeleton-related genes. Among these genes are Aquaporin 1 (Aqp1), Ras-related protein Rab-7a (Rab7), Sodium/potassiumtransporting ATPase gamma chain (Fxyd2), Voltagedependent anion channel 1 (Vdac1), and Guanine nucleotide binding protein, alpha stimulating (Gnas).

\section{Discussion}

The subfamily Sigmodontinae includes about 400 species and 86 living genera [2,3]. Of these, A. olivacea inhabits a wide range of contrasting environments and presents 


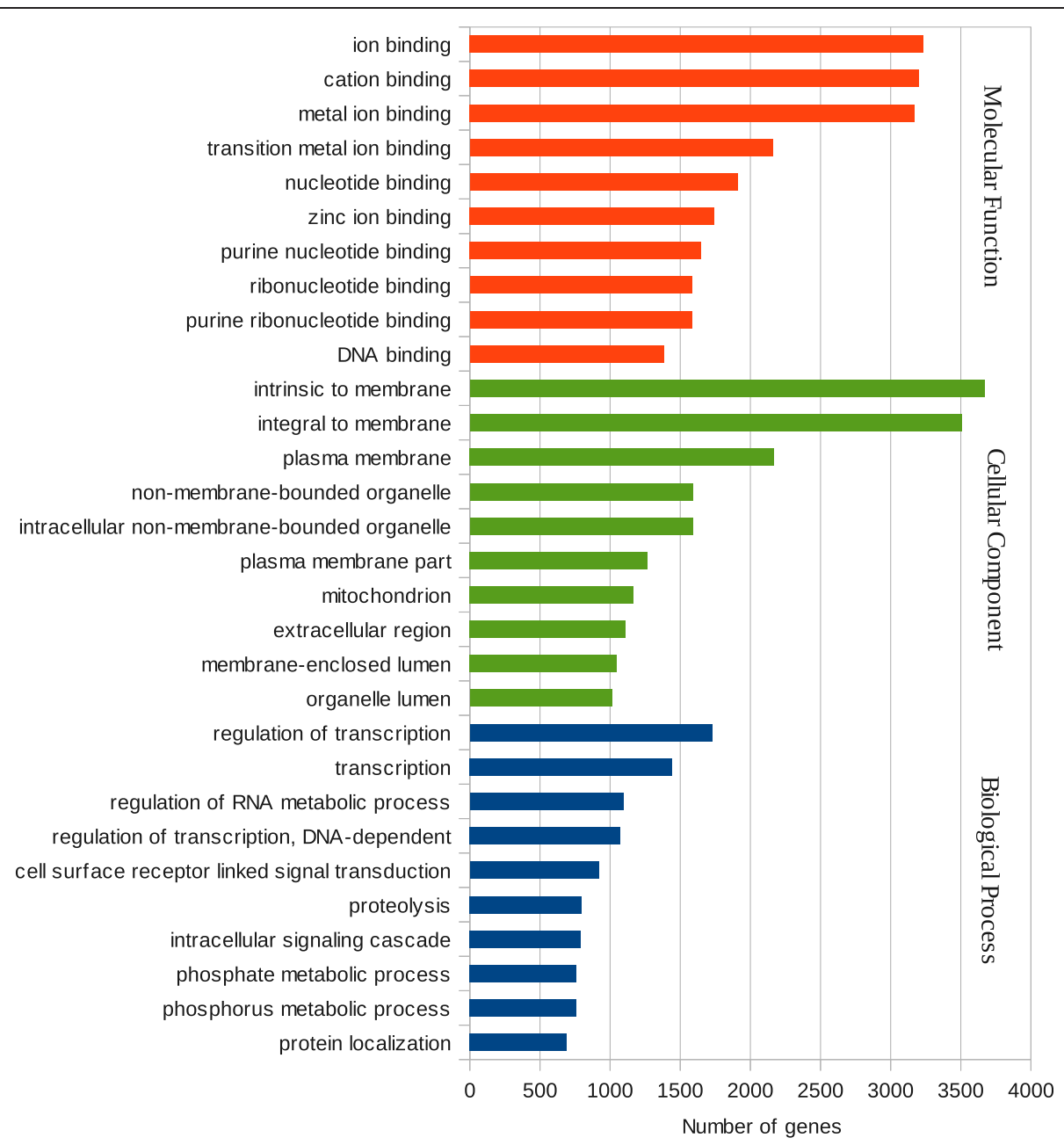

Figure 2 Gene Ontology classification of genes found by TrinityNorm strategy. Results are summarized in three main categories: Biological process, Molecular function, and Celullar component.

different geographic forms. In this work, 13 individuals were sampled in Argentina and Chile, covering both the arid Patagonian steppe and the wet Valdivian and Magellanic forests. More than $800 \mathrm{M}$ reads were generated in what constitutes the first characterization of a sigmodontine transcriptome. In addition, we present a set of highly expressed genes of which some are possible candidates for ecological studies of the response of the species to environmental variation in water and dietary items availability.

\section{Transcriptome sequencing and assembly}

As the cost of high-throughput sequencing falls and more cDNA sequences are generated, the importance of appropriate normalization strategies prior to contig assembling increases. In this study, we used two normalization strategies and compared their performance with a nonnormalized (Multireads) alternative.

In terms of length and number of contigs assembled regardless of the strategy, our results were similar to those found in previous studies in which Trinity was used [31,32]. Among the three strategies, if only contigs descriptive statistics are taken into account, the assembly after both normalization strategies clearly outperformed the Multireads approach. Normalizations not only showed the largest average and mean contig lengths but also ran considerably faster than the Multireads counterpart. This is a consequence of discarding reads that are considered to be redundant and the concomitant sequencing error removal. If transcriptome coverage is moderate, it is necessary to keep in mind that, as noted by Brown et al. [19], the memory requirements will be roughly the same, with or without normalization, due to limited removal of erroneous k-mers.

\section{Gene annotation and evaluation of reconstructed coding sequences}

To evaluate the capacity of each strategy to assembly the A. olivacea transcriptome, we quantified the number of 
contigs that resulted to be homologs of mouse OMA proteins at different reconstruction levels. We found that the Multireads strategy performs the best for the $>99 \%,>90$ and $>80 \%$ of the total mouse CDS length and obtained the most similar values to those found through the union of the individual sets of contigs. Even though the Multireads strategy produced the assembly with smaller median and mean contig sizes, larger contigs were reconstructed (Additional file 1: Table S3), thus explaining the better results obtained for the CDS reconstruction. Therefore, studies that require complete reconstruction of coding sequences should avoid normalization; but obviously, when billions of reads are available for assembly, the Multireads approach becomes prohibitive and normalization is the only way to proceed. These results are consistent with those found by [19]; it seems that normalization generates a more fragmented assembly, at least when Trinity is used. Our results are consistent with the notion that fragmentation is a by-product of normalization, but also that normalization negatively impacts the completeness of the coding sequences reconstruction at all levels. For both normalization methods a $\mathrm{k}=25$ ( $\mathrm{k}$-mer) was set, and it is not clear why such fragmentation is produced.

If assembly capacity and computational requirements are considered, we consider that assembly from TrinityNorm has the best cost-benefit return. This strategy was second after Multireads in number of genes reconstructed for each category while using about $1 / 10$ of the time. Moreover, a high number of putative isoforms were reconstructed, supported by the number of contigs reconstructed per category (Table 2) and the isotigs counted from Trinity assembly (see Results). Even though DigiNorm reached the largest number of isotigs and the highest number of genes, its performance at reconstructing full and almost full CDS was the worst and very similar to the best single library. Also, the assembly from DigiNorm was two times slower than that from TrinityNorm. The latter required more time for normalization but is capable of running multithreading and so outperforms DigiNorm.

Regardless of the strategy, a large number of homologous mouse genes were obtained in our study. According to microarray studies [33], about 7600 genes are expressed in the kidneys of adult humans; meanwhile, recent HTS transcriptome studies found 15,369 for the baboon kidneys [34]. Clearly, in this study we were able to find almost the same number of genes $(15,077$ through TrinityNorm) that Spradling et al. [34] even though we used a very stringent e-value for the BLASTX analysis. Moreover, of those genes, $40 \%$ had at least one full (>99\%) CDS reconstructed. Given that, we reconstructed 8,325 distinct mouse isoforms out of 24,338 ( $\sim 34 \%)$ for at least $90 \%$ of the total expected length, we established an important set of sequences, likely orthologous to mouse genes, which will be useful for future analyses of molecular evolution, population genomics, and phylogenetics.

\section{List of most expressed genes}

The assessment of gene differential expression tends to be problematic for contigs with low counts [35]; therefore, a good strategy is establishing a set of highly expressed genes for directing efforts to study differential expression. In this work, 13 transcriptome libraries were used to identify the most expressed genes of the kidneys of $A$. olivacea. Some of them had already been described for model species, while many of the new ones have a clear relationship with renal function and could serve as potential candidates for future evolutionary and ecological genomic studies.

Seventeen of 283 most expressed genes found in this work were previously singled out by Pradervand et al. [30] using microarrays in the distal part of the mouse renal tubule. Although for some of these genes their precise function is not clear, for others knowledge on their function is reasonably good. For example, Aqp1 is involved in water reabsorption at the apical and basolateral plasma membrane of the proximal tubule [36]; mutations in Fxyd2 have been associated with renal hypomagnesemia2 [37]; Rab7, as a Rab member, could be implicated in the transport, docking, and fusion of endocytotic vesicles [30], and finally, Gnas codifies the alpha subunit of heterotrimeric $G$ proteins, which mediates the vasopressin receptor type 2 signaling after the binding with vasopressin, and ultimately increases water reabsorption in the collecting duct [36]. In our expression analysis, Fxyd2 and Aqp1 are among the top 10 and top 50 of the most expressed genes, respectively. The latter represents a good candidate gene for the study of differential responses to variation of environmental water availability.

Among the 266 remaining highly expressed genes, additional putative candidates associated with renal function were found; for example: i) kallikrein (Klk1) encodes a proteolytic protein which produces the kinin proteins, which may counteract the hydrosmotic effect of vasopressin [36]; ii) Uromodulin (Umod) encodes the most abundant protein in urine [38], and mouse knockouts for this gene have shown urine concentration problems [36]; iii) Glyoxylate reductase (Grhpr), an enzyme that catalyzes the reduction of glyoxylate to glycolate, is associated with a disorder that can cause nephrolithiasis (kidney stone), nephrocalcinosis, and renal failure [39,40]; and iv) Sorbitol Dehydrogenase (Sord), along with Aldose reductase, are possibly involved in osmoregulation in the kidney [36,41].

Regarding the GO-term classification, no important differences were found between the set of all genes and the 283 most expressed ones, except that an expected excess of mitochondrial related GO-terms was found among the latter (Additional file 3: Table S6). This enrichment is not 
surprising as the kidney is an energetically demanding organ $[42,43]$.

\section{Conclusion}

In order to obtain the best-reconstructed transcripts from the kidney of the olive mouse A. olivacea on the basis of 13 individual libraries, we first explored three alternative assembling strategies. Results indicate that the Trinity's in silico normalization is the best algorithm in terms of costbenefit return. We annotated more than 10,000 genes that were almost fully reconstructed, calculated their expression levels, and identified the most expressed ones. Various genes involved in water conservation in mouse models under laboratory conditions were reconstructed and showed high expression levels in A. olivacea, demonstrating the value of RNA-seq technology. Given that this work is the first to characterize the transcriptome of any member of Sigmodontinae, a subfamily of cricetid rodents with about 400 species, it will provide valuable resources for future ecological genomics and evolutionary analyses and will serve as assembly reference for a large number of species. In particular, it will facilitate the study of variation in levels of gene expression in the olive mouse and other sigmodontines that occupy a wide range of environmental conditions-from aridlands to temperate rainforests-in South America.

\section{Methods}

\section{Data collection}

Individuals were collected with Sherman traps from the following localities: Fundo San Martín, Los Ríos $(\mathrm{n}=4)$ and Sector Barrancoso, Aysén $(\mathrm{n}=4)$ in Chile, and Gan Gan, Chubut ( $\mathrm{n}=2)$ and Río Oro, Santa Cruz $(\mathrm{n}=3)$ in Argentina (further details in Additional file 1: Table S1). Kidneys were frozen in liquid nitrogen in the field immediately following euthanization. All steps involving live animals followed the recommendations of Sikes et al. [44].

\section{RNA extraction and library construction}

For each individual, RNA extraction was conducted in one half of the kidney after a lengthwise cut. To this end, the RNeasy mini kit (Qiagen) was employed following recommendations of the manufacturer. RNA quantity and purity was assessed with NanoDrop 1000 Technologies spectrophotometer. RNA integrity was checked through electrophoresis in Formaldehyde-agarose 1,2\% denaturing gels. Libraries were constructed and sequenced at Macrogen (Korea). Poly-A based mRNA enrichment method and paired-ends library preparation were done following the Illumina TruSeqTM RNA sample preparation kit, according to the instructions of the manufacturer. Library sequencing was performed on Illumina HiSeq 2000 platform.

\section{De novo transcriptome assembly}

Assembly was carried out using default Trinity settings, after removing low quality reads, filtering adaptors and primers, and trimming the 3 ' ends of reads with a quality less than 24 $(\mathrm{Q}<24)$ with FASTX-Toolkit (http:// hannonlab.cshl.edu/fastx_toolkit/). Quality control was checked by FastQC (http://www.bioinformatics.babraham. ac.uk/projects/fastqc/). All assemblies were done on the same single node-machine with $256 \mathrm{G}$ memory and 4 Intel Xeon CPU E7-8837 (8 core) processors. In order to obtain a more complete set of the genes expressed, we pooled individuals from different points of the species distribution and analyzed three strategies: i) merge the reads of the 13 libraries (Multireads) ii) Trinity in silico read normalization (TrinityNorm v2013-08-15), and iii) digital normalization (DigiNorm) with khmer (0.8.2). TrinityNorm and DigiNorm were ran on the same computer (Intel Core i7-3820 processor). Normalization algorithms were designed to systematize the coverage in shotgun sequencing data sets, thereby removing redundant reads. As a consequence, computational requirements are reduced, supposedly without negatively impacting assembly quality. For TrinityNorm the default commands were run with a max coverage (max_cov) of 30. Before running DigiNorm, reads were shuffled and a kmer length of 25 and coverage of 30 were specified. We trimmed off likely erroneous k-mers with the filter-abund.py script. For each assembly we tracked the runtimes.

\section{Gene annotation and GO-terms assignment}

BLASTX (e-value cut offs $<1 \mathrm{e}-10$ ) searches were performed against OMA browser mouse proteins and NCBI non-redundant vertebrate protein databases. Search against OMA browser database is a cost-effective way of gene annotation and allows an upper bound estimation of genes orthologous to those of the mouse among the reconstructed contigs. This database contains most or all exons of a given gene, keeping the number of sequences as low as possible. The longest variant is always retained; shorter variants are also kept if they differ by at least in $10 \%$ of their sequence from the longer variants retained.

For the BLASTX analysis we report, i) the number of contigs that overlap the proteins of mouse genes at $>$ $99 \%,>90 \%,>80 \%$ and $>50 \%$ of their length; ii) the number of distinct mouse proteins reconstructed by a putatively homologous contig using those cutpoints; and iii) the number of distinct genes found with at least one putatively homologous contig reconstructing the gene CDS at or above those percentages. We also reported, the number of contigs, coding sequences, and genes that had a significant hit (e-value $<1 \mathrm{e}-10)$ independently of the alignment proportion. To report the number of genes, contigs annotated as putatively homologs to 
mouse OMA proteins entries were grouped into the corresponding mouse genes using the oma-ensembl file at OMA-Browser webpage through in-house-scripts (Additional file 4).

The contigs assembled from the Multireads, TrinityNorm and DigiNorm strategies, were annotated for protein domains through the Pfam database using HMMER. An e-value threshold of 1e-2 was set. Before running this analysis we first predicted the exon/intron structure of each contig using the software Augustus [45] trained with Homo sapiens. This software has been used extensively for gene prediction (e.g. [46-48]). The GTF files from Augustus output are available upon request. Only the nonoverlapping protein domains found were reported on the summary file of BLASTX and Pfam results. A in-housescript was used for this purpose (Additional file 4).

The average number of potential isoforms reconstructed from the Trinity assembly was calculated averaging the times that a particular "comp_XXX" (as given by the ID of Trinity assembled contig) is repeated.

Gene Ontology analysis was done using the DAVID bioinformatics database, using the Benjamini correction of $p<0.05$ as criterion for enrichment. First, we classify the most common GO-terms from the genes list obtained from the TrinityNorm assembly. Then, we used that gene list as background for analyzing the ontology of the most expressed genes.

\section{List of most expressed genes}

To determine the most expressed genes in the kidneys of $A$. olivacea, we sought for genes that were in common among the $5 \%$ most expressed genes in each of the 13 transcriptomes. Despite this being a very conservative approach, it was preferred because it would generate a reliable list of genes.

To this end, we first aligned RNA-Seq reads in a paired end fashion against each reference transcript using the aligner Bowtie [49]. Then, we calculated genelevel TPM values using RSEM (v1.2.4). The results of BLASTX for each transcriptome against mouse OMA browser protein, and the OMA-ensembl corresponding pair were used to specify which transcripts were from the same gene. This program hands reads that map to multiple transcripts avoiding throwing away data and biased estimates without relying on the existence of a reference genome. Finally, bash commands and in-house scripts (Additional file 4) were used to obtain the most expressed genes as described above.

\section{Availability of supporting data}

The sequencing data has been deposited to the Sequence Read Archive database (accession number SRP033780).

\section{Animal ethics statements}

All methods involving A. olivacea were carried out in accordance with a protocol reviewed and approved by the Ethics Committee of the Fondo Nacional de Ciencia y Tecnología (FONDECYT, Chile) and the Ethics Committee of the Universidad Austral de Chile (UACh, Chile), as part of the review process for the Fondecyt Research Grant 1110737.

\section{Additional files}

\begin{abstract}
Additional file 1: Sampling localities, assembly metrics, contig distribution and gene annotation. Table S1. Specimen ID numbers, sampling localities, and read lengths before and after trimming. Table S2. Descriptive statistics of individual RNA-seq samples and reconstructions. Table S3. Distribution of contig sizes and their relative proportions for each assembly protocol. Table S4a. Gene annotation and CDS reconstruction using BLASTX to OMA browser mouse protein database. Table S4b. Annotation using BLASTX to NCBI non-redundant vertebrate protein database. Table S5. Annotation through Pfam database using HMMER.

Additional file 2: Summarizing the BLASTX and Pfam results for Multireads, TrinityNorm and DigiNorm strategies.

Additional file 3: List of the $\mathbf{2 8 3}$ most expressed genes and its GO-terms classification. Table S5. List of 283 genes represented in the $5 \%$ most expressed genes of each of the individual samples. Table S6. Gene Ontology classification of the 283 most expressed genes.

Additional file 4: Containing the in-house-python scripts.
\end{abstract}

\section{Abbreviations}

GO: Gene ontology; CDS: Coding sequences; TrinityNorm: Trinity in silico normalization; DigiNorm: Digital normalization.

\section{Competing interests}

The authors declare that they have no competing interests.

\section{Authors' contributions}

MF, GD, LV, DEN, and EPL participated in the field work and sample preparations. W did the RNA preparations. FG, MF, and LV carried out the analyses. GD, JCO, DEN, and EPL designed and supervised the project. FG wrote the manuscript. All authors approved the final manuscript.

\section{Acknowledgments}

We are grateful to Ulyses Pardiñas, Pablo Teta, Daniel Udrizar-Sauthier Mauro Tammone, and Ivanna Tomasco for assistance in the field. We thank Matthew MacManes and Joseph Cook for comments on an earlier version of the manuscript, and the Bioinformatics Unit, Institut Pasteur de Montevideo, for computational support. Work was supported by grants from CSIC-Universidad de la República, Agencia Nacional de Investigación e Innovación (ANII), Uruguay, and FONDECYT 1110737, Chile. Facundo Giorello and Matías Feijoo are supported by graduate fellowships from ANII.

\section{Author details}

${ }^{1}$ Departamento de Ecología y Evolución, Facultad de Ciencias, Universidad de la República, Montevideo, Uruguay. ${ }^{2}$ Instituto de Ciencias Ambientales y Evolutivas, Universidad Austral de Chile, Valdivia, Chile.

Received: 13 December 2013 Accepted: 27 May 2014

Published: 8 June 2014

\section{References}

1. Waterhouse GR: Characters of new species of the genus Mus, from collection of Mr. Darwin. Proc Zool Soc London 1837, 5:15-21. 27-32.

2. D'Elía G, Pardiñas UFJ, Teta P, Patton JL: Definition and diagnosis of a new tribe of sigmodontine rodents (Cricetidae: Sigmodontinae), and a revised classification of the subfamily. Gayana 2007, 71:187-194. 
3. D'Elía G, Pardiñas UFJ: Subfamily Sigmodontinae Wagner, 1843. In Mamm South Am Vol 2 Rodents. Edited by Patton JL, Pardiñas UFJ, D'Elía G. Chicago: University of Chicago Press; in press.

4. Mann G: Los pequeños mamíferos de Chile. Gayana Zool 1978, 40:1-342.

5. Pizarro R, Valdés R, García-chevesich P, Vallejos C, Sangüesa C, Morales C, Balocchi F, Abarza A, Fuentes R: Latitudinal analysis of rainfall intensity and mean annual precipitation in chile. Chil J Agric Res 2012, 72:252-261.

6. Bozinovic F, Rojas JM, Gallardo PA, Palma RE, Gianoli E: Body mass and water economy in the South American olivaceous field mouse along a latitudinal gradient: Implications for climate change. J Arid Environ 2011, 75:411-415

7. Rodríguez-Serrano E, Cancino R a, Palma RE: Molecular phylogeography of Abrothrix olivaceus (Rodentia: Sigmodontinae) in Chile. J Mammal 2006, 87:971-980

8. Lessa EP, D'Elía G, Pardiñas UFJ: Genetic footprints of late Quaternary climate change in the diversity of Patagonian-Fueguian rodents. Mol Ecol 2010, 19:3031-3037

9. Metzker ML: Sequencing technologies - the next generation. Nat Rev Genet 2010, 11:31-46.

10. Rizzo JM, Buck MJ: Key principles and clinical applications of "nextgeneration" DNA sequencing. Cancer Prev Res 2012, 5:887-900.

11. Morozova O, Marra M a: Applications of next-generation sequencing technologies in functional genomics. Genomics 2008, 92:255-264.

12. Ekblom R, Galindo J: Applications of next generation sequencing in molecular ecology of non-model organisms. Heredity (Edinb) 2011, 107:1-15.

13. Shokralla S, Spall JL, Gibson JF, Hajibabaei M: Next-generation sequencing technologies for environmental DNA research. Mol Ecol 2012, 21:1794-1805.

14. Garg R, Patel RK, Tyagi AK, Jain M: De novo assembly of chickpea transcriptome using short reads for gene discovery and marker identification. DNA Res 2011, 18:53-63.

15. Shi C-Y, Yang H, Wei C-L, Yu O, Zhang Z-Z, Jiang C-J, Sun J, Li Y-Y, Chen Q, Xia T, Wan X-C: Deep sequencing of the Camellia sinensis transcriptome revealed candidate genes for major metabolic pathways of tea-specific compounds. BMC Genomics 2011, 12:131.

16. Lorizzo M, Senalik DA, Grzebelus D, Bowman M, Cavagnaro PF, Matvienko M, Ashrafi $H$, Van Deynze A, Simon PW: De novo assembly and characterization of the carrot transcriptome reveals novel genes, new markers, and genetic diversity. BMC Genomics 2011, 12:389.

17. Feldmeyer B, Wheat CW, Krezdorn N, Rotter B, Pfenninger M: Short read Illumina data for the de novo assembly of a non-model snail species transcriptome (Radix balthica, Basommatophora, Pulmonata), and a comparison of assembler performance. BMC Genomics 2011, 12:317.

18. Wang Z, Gerstein M, Snyder M: RNA-Seq: a revolutionary tool for transcriptomics. Nat Rev Genet 2009, 10:57-63.

19. Brown CT, Howe A, Zhang Q, Pyrkosz AB, Brom TH: A reference-free algorithm for computational normalization of shotgun sequencing data 2012, arXiv:1203.4802v2 [q-bio.GN].

20. Haas BJ, Papanicolaou A, Yassour M, Grabherr M, Blood PD, Bowden J, Couger MB, Eccles D, Li B, Lieber M, Macmanes MD, Ott M, Orvis J, Pochet N, Strozzi F, Weeks N, Westerman R, William T, Dewey CN, Henschel R, Leduc RD, Friedman N, Regev A: De novo transcript sequence reconstruction from RNA-seq using the Trinity platform for reference generation and analysis. Nat Protoc 2013, 8:1494-1512.

21. Mbassa GK: Mammalian renal modifications in dry environments. Vet Res Commun 1988, 12:1-18.

22. Shultz PJ, Tolins JP: Adaptation to increased dietary salt intake in the rat Role of endogenous nitric oxide. J Clin Invest 1993, 91:642-650.

23. Grabherr MG, Haas BJ, Yassour M, Levin JZ, Thompson DA, Amit I, Adiconis X, Fan L, Raychowdhury R, Zeng Q, Chen Z, Mauceli E, Hacohen N, Gnirke A, Rhind N, Di Palma F, Birren BW, Nusbaum C, Lindblad-Toh K, Friedman N, Regev A: Full-length transcriptome assembly from RNA-Seq data without a reference genome. Nat Biotechnol 2011, 29:644-652.

24. Altenhoff AM, Schneider A, Gonnet GH, Dessimoz C: OMA 2011: orthology inference among 1000 complete genomes. Nucleic Acids Res 2011, 39:D289-D294.

25. Finn RD, Mistry J, Tate J, Coggill P, Heger A, Pollington JE, Gavin OL Gunasekaran P, Ceric G, Forslund K, Holm L, Sonnhammer ELL, Eddy SR, Bateman A: The Pfam protein families database. Nucleic Acids Res 2010 38:D211-D222.
26. Eddy SR: Profile hidden Markov models. Bioinformatics 1998, 14:755-763.

27. Eddy SR, Mitchison G, Durbin R: Maximum discrimination hidden Markov models of sequence consensus. J Comput Biol 1995, 2:9-23.

28. Dennis G, Sherman BT, Hosack DA, Yang J, Gao W, Lane HC, Lempicki RA: DAVID: Database for Annotation, Visualization, and Integrated Discovery. Genome Biol 2003, 4:P3.

29. Li B, Dewey CN: RSEM: accurate transcript quantification from RNA-Seq data with or without a reference genome. BMC Bioinforma 2011, 12:323.

30. Pradervand S, Zuber Mercier A, Centeno G, Bonny O, Firsov D: A comprehensive analysis of gene expression profiles in distal parts of the mouse renal tubule. Pflügers Arch Eur J Physiol 2010, 460:925-952.

31. Hershkovitz V, Sela N, Taha-Salaime L, Liu J, Rafael G, Kessler C, Aly R, Levy M, Wisniewski M, Droby S: De-novo assembly and characterization of the transcriptome of Metschnikowia fructicola reveals differences in gene expression following interaction with Penicillium digitatum and grapefruit peel. BMC Genomics 2013, 14:168.

32. Liu T, Zhu S, Tang Q, Chen P, Yu Y, Tang S: De novo assembly and characterization of transcriptome using Illumina paired-end sequencing and identification of CesA gene in ramie (Boehmeria nivea L. Gaud). BMC Genomics 2013, 14:125.

33. Yano $N$, Endoh M, Fadden $K$, Yamashita $H$, Kane A, Sakai $H$, Rifai A: Comprehensive gene expression profile of the adult human renal cortex: analysis by cDNA array hybridization. Kidney Int 2000, 57:1452-1459.

34. Spradling KD, Glenn JP, Garcia R, Shade RE, Cox LA: The baboon kidney transcriptome: analysis of transcript sequence, splice variants, and abundance. PLoS One 2013, 8:e57563.

35. Bullard JH, Purdom E, Hansen KD, Dudoit S: Evaluation of statistical methods for normalization and differential expression in mRNA-Seq experiments. BMC Bioinforma 2010, 11:94.

36. Fenton RA, Knepper MA: Mouse models and the urinary concentrating mechanism in the new millennium. Physiol Rev 2007, 87:1083-1112.

37. Meij IC, Koenderink JB, Van Bokhoven H, Assink KF, Groenestege WT, De Pont JJ, Bindels RJ, Monnens LA, Van Den Heuvel LP, Knoers NV: Dominant isolated renal magnesium loss is caused by misrouting of the $\mathrm{Na}+$, K+-ATPase gamma-subunit. Ann N Y Acad Sci 2003, 986:265-266.

38. Bachmann S, Dawnay AB, Bouby N, Bankir L: Tamm-Horsfall protein excretetion during chronic alterations in urinary concentration and protein intake in rat. Ren Physiol Biochem 1991, 14:236-245.

39. Cramer SD, Ferree PM, Lin K, Milliner DS, Holmes RP: The gene encoding hydroxypyruvate reductase (GRHPR) is mutated in patients with primary hyperoxaluria type II. Hum Mol Genet 1999, 8:2063-2069.

40. Lam CW, Yuen YP, Lai CK, Tong SF, Lau LK, Tong KL, Chan YW: Novel mutation in the GRHPR gene in a Chinese patient with primary hyperoxaluria type 2 requiring renal transplantation from a living related donor. Am J Kidney Dis 2001, 38:1307-1310.

41. Steffgen J, Kampfer K, Grupp C, Langenberg C, Müller GA, Grunewald RW: Osmoregulation of aldose reductase and sorbitol dehydrogenase in cultivated interstitial cells of rat renal inner medulla. Nephrol Dial Transplant 2003, 18:2255-2261.

42. Al Samri MT, Al Shamsi M, Al-Salam S, Marzouqi F, Al Mansouri A, Al-Hammadi S, Balhaj G, Al Dawaar SKM, Al Hanjeri RSMS, Benedict S, Sudhadevi M, Conca W, Penefsky HS, Souid A-K: Measurement of oxygen consumption by murine tissues in vitro. J Pharmacol Toxicol Methods 2011, 63:196-204

43. Tahara EB, Navarete FDT, Kowaltowski AJ: Tissue-, substrate-, and site-specific characteristics of mitochondrial reactive oxygen species generation. Free Radic Biol Med 2009, 46:1283-1297.

44. Sikes RS, Gannon WL: Guidelines of the American Society of Mammalogists for the use of wild mammals in research. J Mammal 2011, 92:235-253.

45. Stanke M, Keller O, Gunduz I, Hayes A, Waack S, Morgenstern B: AUGUSTUS: ab initio prediction of alternative transcripts. Nucleic Acids Res 2006, 34:W435-W439.

46. Cornman R, Bennett AK, Murray K, Evans JD, Elsik CG, Aronstein K. Transcriptome analysis of the honey bee fungal pathogen. Ascosphaera apis: implications for host pathogenesis. BMC Genomics 2012, 13:285

47. Wang S, Furmanek T, Kryvi H, Krossøy C, Totland GK, Grotmol S, Wargelius A: Transcriptome sequencing of Atlantic salmon (Salmo salar L.) notochord prior to development of the vertebrae provides clues to regulation of positional fate, chordoblast lineage and mineralisation. BMC Genomics 2014, 15:141. 
48. Eckalbar WL, Hutchins ED, Markov GJ, Allen AN, Corneveaux JJ, Lindblad-Toh K, Di Palma F, Alföldi J, Huentelman MJ, Kusumi K: Genome reannotation of the lizard Anolis carolinensis based on 14 adult and embryonic deep transcriptomes. BMC Genomics 2013, 14:49.

49. Langmead B, Trapnell C, Pop M, Salzberg SL: Ultrafast and memory-efficient alignment of short DNA sequences to the human genome. Genome Biol 2009, 10:R25.

doi:10.1186/1471-2164-15-446

Cite this article as: Giorello et al:: Characterization of the kidney

transcriptome of the South American olive mouse Abrothrix olivacea. BMC Genomics 2014 15:446.

\section{Submit your next manuscript to BioMed Central and take full advantage of:}

- Convenient online submission

- Thorough peer review

- No space constraints or color figure charges

- Immediate publication on acceptance

- Inclusion in PubMed, CAS, Scopus and Google Scholar

- Research which is freely available for redistribution 